\title{
PREGNANCY COMPLICATED BY ACUTE INTESTINAL OBSTRUCTION SECONDARY TO LEFT PARADUODENAL HERNIA- AN UNUSUAL CASE REPORT
}

\author{
Sumbul Ali1 ${ }^{1}$ Bharti Saxena ${ }^{2}$, Ram Gopal Meena ${ }^{3}$, Anand Vijay4, Rajendra Kumar Tanwar ${ }^{5}$ \\ ${ }^{1}$ Assistant Professor, Department of Obstetrics \& Gynaecology, Government Medical College, Kota. \\ 2 Professor, Department of Obstetrics \& Gynaecology, Government Medical College, Kota. \\ ${ }^{3}$ Assistant Professor, Department of Surgery, Government Medical College, Kota. \\ ${ }^{4}$ Senior Resident Department of Surgery, Government Medical College, Kota. \\ 5 Professor, Department of Radiotherapy, Government Medical College, Kota.
}

\section{ABSTRACT}

\section{BACKGROUND}

A 25-year-old female with 34 weeks' gestation and intestinal obstruction was diagnosed to have paraduodenal hernia during laparotomy. Paraduodenal hernia is a congenital anomaly where the small intestine is trapped in the internal hernial sac. Only if high suspicion is kept in mind, this rare entity may be diagnosed preoperatively. All modern imaging modalities including USG, CT scan and MRI suggest only intestinal obstruction along with gravid uterus.

\section{KEYWORDS}

Pregnancy, Left Paraduodenal Hernia, Intestinal Obstruction.

HOW TO CITE THIS ARTICLE: Ali S, Saxena B, Meena RG, et al. Pregnancy complicated by acute intestinal obstruction secondary to left paraduodenal hernia- An unusual case report. J. Evolution Med. Dent. Sci. 2017;6(4):340-342, DOI: 10.14260/Jemds/2017/76

\section{BACKGROUND}

Internal hernias are a rare cause of intestinal obstruction. Paraduodenal hernias (PDH) account for $50 \%$ of intestinal obstruction due to internal hernias.[1] The average age of presentation is 38.5 years.[1] $\mathrm{PDH}$ are 3 times more common in males than females. Left-sided PDH is 3 times more common than right-sided PDH. Knowledge of this entity, anatomy, associated symptoms and timely surgical treatment can reduce the morbidity and mortality associated with this condition. Hundreds of cases of PDH causing intestinal obstruction have been reported earlier in literature, but only one case of PDH associated with pregnancy has been reported by Chan $\mathrm{HM}$ et al.[2] We are hereby presenting a similar rare case of acute intestinal obstruction secondary to LPDH in pregnancy at 36 weeks.

\section{Case Report}

A 25-year-old primigravida presented to the obstetric department in emergency with complaints of term pregnancy and acute abdominal pain and vomiting since 1 day. She gave history of nausea, retching and indigestion throughout the pregnancy. On examination, patient appeared dehydrated and had an anxious and toxic look. Her pulse was $120 /$ minute, BP - 100/70 mmHg and Temperature- 370 Celsius. On clinical examination, abdomen was distended up to xiphisternum with gravid uterus of 34 weeks size. On careful clinical examination, a separate cystic swelling was seen above the gravid uterus, occupying whole of the left hypochondrium, epigastrium and some part of right hypochondrium. The swelling was tense, tender and guarding was present. The uterus was irritable with foetus in cephalic

Financial or Other, Competing Interest: None.

Submission 25-11-2016, Peer Review 30-12-2016,

Acceptance 05-01-2017, Published 12-01-2017.

Corresponding Author:

Dr. Bharti Saxena,

\#B-26, Sukhdham Colony,

Police Lines, Baran Road,

Kota-324001, Rajasthan.

E-mail: bharti.saxena.kota@gmail.com

DOI: $10.14260 /$ jemds $/ 2017 / 76$ presentation. The foetal heart sounds were not audible. On per vaginal examination, the findings were suggestive of latent phase of labour. Blood tests were sent and conservative measures were started to correct dehydration. A nasogastric tube was inserted to decompress the distended abdomen and resulted in flow of thick dirty yellow coloured aspirate of $800 \mathrm{~mL}$. USG showed features of subacute intestinal obstruction with mild-to-moderate haemoperitoneum. Obstetric scan showed intrauterine foetal demise. Needling under USG guidance confirmed haemoperitoneum. The X-ray of abdomen in sitting position showed dilated bowel loops and no signs of perforation of gut. Blood tests revealed leukocytosis (TLC- 21000/cu.mm) with neutrophilia (85\%). Serum sodium was reduced (128 nmol/L).

After taking consent, an emergency laparotomy was undertaken in collaboration with general surgeon. The abdomen was opened by midline incision. Haemoperitoneum about 200 cc was seen. LSCS was done. A dead female child, weighing $2.5 \mathrm{~kg}$ weight was extracted. The liquor was clear and there was no post-partum haemorrhage. The uterus was closed in two layers. On further exploration of upper abdomen after extending the subumbilical incision up to xiphisternum. A mass was seen of $25 \mathrm{~cm} \mathrm{x} 20 \mathrm{~cm}$ superior to transverse colon and covered by omentum in lesser sac. Caecum and ascending colon were found to be mobile and were found drawn up to the left hypochondrium. The exploration of the mass by surgeon revealed most of the jejunum and ileum herniated, through the left paraduodenal hernia site measuring $5 \mathrm{~cm}$ x $5 \mathrm{~cm}$ size, into left paraduodenal fossa. The hernial sac was dilated, taking care of the inferior mesenteric vessels to deliver the herniated small bowel. Approximately, 5 metres of small bowel was found gangrenous and was therefore resected. About one foot of jejunum and 1 foot of ileum were found viable, which were brought out as 2 separate stoma out of the abdominal wall. The mobile caecum was later fixed to the right iliac fossa peritoneum. On sixth post-operative day, the proximal jejunal stoma became gangrenous and therefore second emergency surgery was done to excise the gangrenous jejunal stoma. For the remaining viable part which was $4 \mathrm{~cm}$ long, jejunoileal 
anastomosis was done. The post-operative period was uneventful and the patient was discharged.

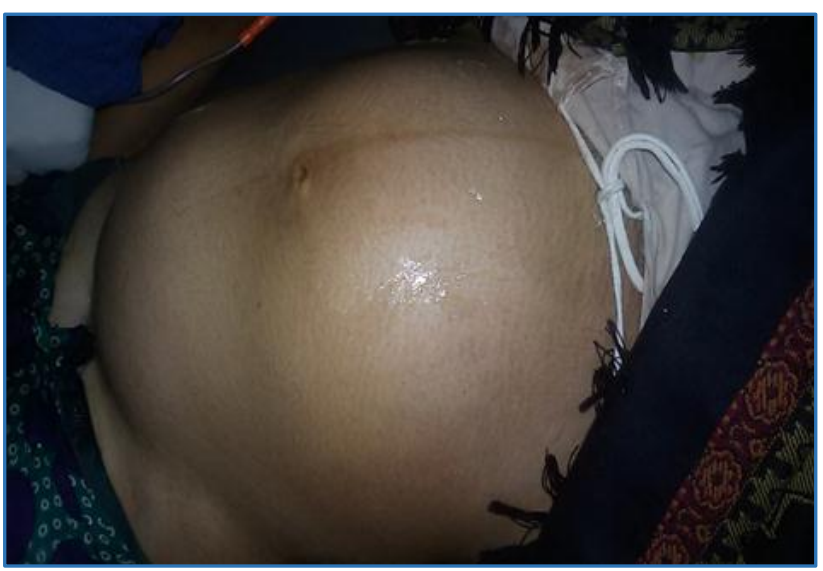

Figure 1. Shows a swelling in upper abdomen separate from gravid uterus

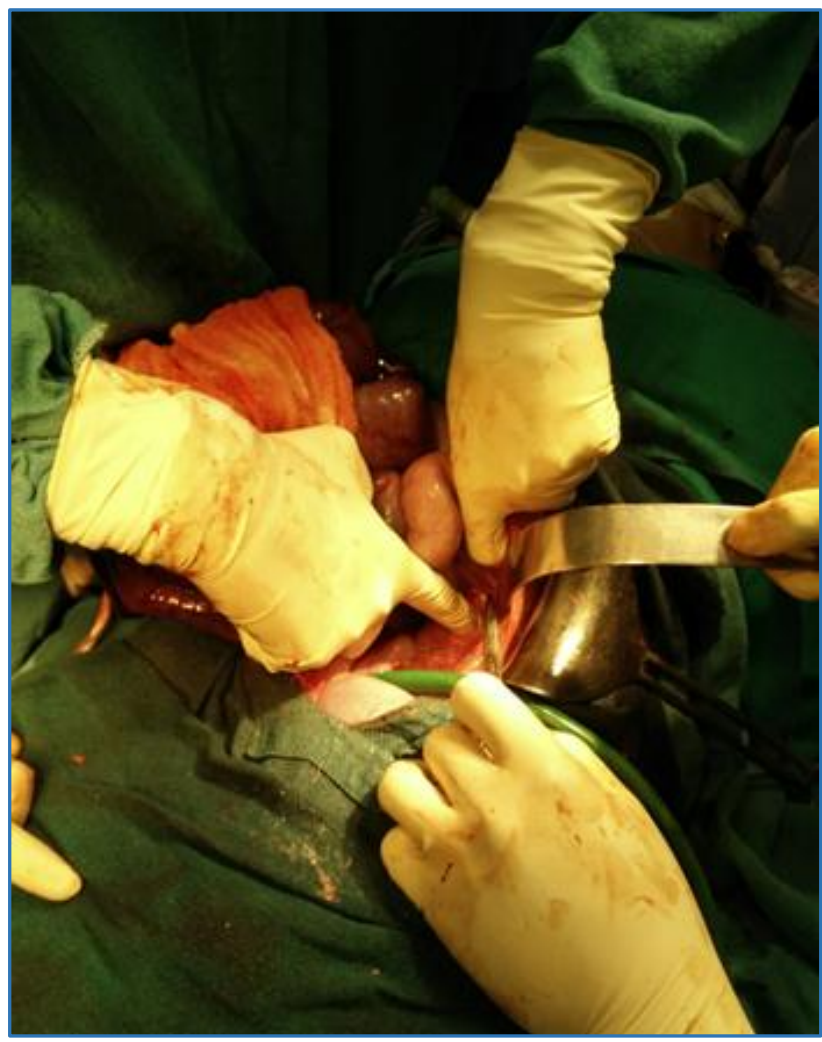

Figure 2. Shows post-caesarean uterus, internal hernia orifice shown by the point of artery forceps, the inferior mesenteric vessels in the anterior wall of the sac and the gangrenous small bowel loops

\section{DISCUSSION}

Internal hernia is defined as a protrusion or bulging of viscus through an opening in the peritoneum or mesentery within the abdominal cavity. The incidence of internal hernia found to be less than $1 \% .{ }^{[2]}$ The internal hernias can be congenital or acquired whereas paraduodenal hernias are the most common congenital internal hernias with an incidence of $53 \%$ of internal hernias.[1] The PDH are of two types - right and left-sided PDH. Left PDH is 3 times more common than Right PDH.

Mechanism of development of LPDH (Proposed by Andrews, 1923)- During embryological development, there is abnormal rotation of midgut (Dorsal instead of ventral) while migrating into the peritoneal cavity, along with failure of fusion of its mesentery with the parietal peritoneum of the posterior abdominal wall, thus leaving a defect (Hernia orifice). In this situation, the inferior mesenteric vessels and left colic artery lies anteriorly to left PDH.[2,3]

With LPDH, there is a $50 \%$ lifetime risk of small bowel obstruction,[3] with mortality reaching $20 \%$ due to small bowel incarceration, strangulation and perforation.

The symptoms associated with LPDH are vague abdominal pain to those of sub-acute or acute intestinal obstruction. Rarely patient presents with acute abdomen with an abdominal mass. Based on symptomatology, LPDH is difficult to diagnose in pregnant women, as abdominal pain, vomiting, obstipation and distention are also found in the course of a normal pregnancy.[2]

Physical examination might be unrevealing or might show nonspecific signs as abdominal tenderness, distention and occasional presence of a mass. ${ }^{[1,4]}$ The enlarged uterus often prevents identification of a separate hernial mass during examination, a growing uterus might distort the hernia sac and might convert a partial intestinal obstruction into a complete one.[2]

Plain abdominal radiograph may show fluid filled stomach or dilated bowel loops of small intestine in an ovoid mass, lateral from midline. Small bowel follow through radiographs may show small bowel herniated through the defect in left upper quadrant of the abdomen.

Ultrasound may show cluster of small bowel loops, dilated stomach, presence or absence of peristalsis and sometimes abdominal mass. CT scan is considered the initial tool of investigation. Findings are abnormal cluster or sac like mass of dilated small bowel loops lying between the pancreas and stomach (Left to the ligament of Treitz). The IMVs appear engorged and crowded above the encapsulated bowel loops.

MRI reveals a mass of herniated small bowel loops in sagittal plane, but is not a cost effective investigation. Treatment of LPDH requires surgery which consists of reduction of herniated small bowel loops followed by either closure of the hernia orifice with non-absorbable suture or an alternative approach is of widening the hernia orifice through an incision along the avascular area of the hernia sac, taking care of preventing injury to IMVs which lie anteriorly to the hernia orifice.

\section{CONCLUSION}

PDH should be considered in the differential diagnosis of young patients with intestinal obstruction or repetitive obstructive symptoms, without history of prior abdominal surgery. Nausea and vomiting are common symptoms during $1^{\text {st }}$ trimester of pregnancy, but if these develop later in pregnancy, other causes should be considered.[2] Once diagnosis is made, surgical treatment should be prompt in order to prevent strangulation of the gut and improve patient outcome.

\section{Declaration}

The manuscript has not been published or submitted for publication elsewhere. All authors have contributed significantly and all the authors are in agreement with the content of the manuscript. Our study does not violate the policies and/or procedures established by journal such as 
those described in "Specific Inappropriate Acts in Publication Process".

\section{Informed Consent}

Patient consent was obtained prior to the study and patient identity not disclosed.

\section{REFERENCES}

[1] Huang YM, Chou AS, Wu YK, et al. Left paraduodenal hernia presenting as recurrent small bowel obstruction. World Journal of Gastroenterology 2005;11(41):6557-9.
[2] Chan HM, Huang TJ, Hsieh JS. Left paraduodenal hernia complicating pregnancy- a case report. Kaohsiung J Med Sci 1990;6:674-7.

[3] Mozaffar M, Hasani M, Fallah M, et al. A left paraduodenal hernia causing bowel obstruction: a case report. Gasteroenterology and Hepatology from Bed to Bench 2013;6(1):48-51.

[4] Kuzinkovas V, Haghighi K, Singhal R, et al. Paraduodenal hernia: a rare cause of abdominal pain. Canadian Journal of Surgery 2008;51(6):E127-8. 